(C) 2016 IEEE. Personal use of this material is permitted. Permission from IEEE must be obtained for all other

uses, in any current or future media, including reprinting/republishing this material for advertising or promotional purposes, creating new collective works, for resale or redistribution to servers or lists, or reuse of any copyrighted component of this work in other works.

\title{
Impairment-aware optimization strategies to dimension optical transport networks with minimal regeneration requirements
}

\author{
Ricardo Mendes*, Bruna Nogueira ${ }^{\dagger}$, Teresa Gomes ${ }^{\ddagger}$, Lúcia Martins ${ }^{\S}$ and João Santos ${ }^{\Uparrow}$ \\ ${ }^{*} \dagger \ddagger$ Department of Electrical and Computer Engineering \\ University of Coimbra, Pinhal de Marrocos 3030-290 Coimbra \\ Email: *rmendes@student.uc.pt, †ban@ student.uc.pt, †teresa@deec.uc.pt and §lucia@deec.uc.pt \\ $\ddagger \S$ Institute for Systems Engineering and Computers at Coimbra \\ Pólo II, R. Silvio Lima, 3030-290 Coimbra \\ ${ }^{\top}$ Coriant Portugal \\ Rua Irmãos Siemens, 1-1A 2720-093 Amadora \\ Email: joao.m.santos@coriant.com
}

\begin{abstract}
Physical impairments restrain the maximum length a signal can travel without regeneration. The quality of a signal in optical wavelength division multiplexing (WDM) networks must thus be restored with opto-electro-optical (OEO) regeneration in order to reach its destination. As OEO regenerators are costly devices, sparse deployment using routing optimization is the key to reduce the network cost. This paper tackles the problem of impairment aware routing and wavelength assignment with regeneration placement (RWARP), considering capacity constraints, while focusing on minimizing the number of regenerators. We will firstly, extend an Integer Linear Programming (ILP) formulation for the RWARP problem which provides, when feasible, an optimal solution, and secondly, propose an efficient heuristic. Results show that the heuristic provides satisfactory results, optimal for small problems, in a fraction of the ILP execution time.
\end{abstract}

\section{INTRODUCTION}

Optical wavelength division multiplexing (WDM) networks have been established as the backbone networks to face the increase in bandwidth demand of today's applications. In this type of networks, the signals travel through lightpaths which may span multiple consecutive fibres on a given wavelength. Physical impairments in the fibres degrade the signal quality as the propagation distance increases. This degradation occurs at the wavelength level and may lead to unacceptable bit error rates (BER). In order to recover its quality, regeneration at the network nodes may be needed so that the signal reaches its destination with a BER below a given threshold. Lightpaths that have regeneration at the intermediate nodes (and consequently conversion to the electrical domain) are referred to as translucent lightpaths as opposed to transparent lightpaths that are unregenerated segments. Optical networks regenerators regenerate the signal per wavelength and thus a node may have multiple regenerators. Opto-electro-optical(OEO) regenerators are still the practical choice as all-optical regenerators are still under development [1], [2]. Since OEO regenerators are expensive devices (CAPEX) and have an high power consump- tion (OPEX), minimizing the number of these elements in the network greatly reduces the network costs. The objective is thus to route the network traffic in a manner that the minimum number of regenerators is needed. Many strategies for Impairment Aware Routing and Wavelength Assignment and Regenerator Placement (IA-RWARP) have been proposed, in order to minimize their deployment throughout the network, where single [3]-[5] and multiple [6]-[10] impairment metrics were taken into consideration.

The contribution of this paper is an extension and modification of the ILP formulation in [5], where we introduced capacity and bidirectional traffic considerations and wavelength conversion capabilities in the network, without considering path protection. To tackle the RWARP problem for large networks, we also extend the heuristic Exact Single Request Regenerator Placement (ESRRP) in [6] to take into account the added constraints of the problem. The networks used on the study were backbone networks and thus the demands will be considered bidirectional symmetric. We will also assume that the regenerators are bidirectional devices. Results are presented for several networks showing the effectiveness of the presented heuristic.

The remainder of the paper is organized as follows. In Section II we present a brief review on the RWARP problem. In Section III we present our ILP formulation and the heuristic formulation. In Section IV we show our simulation data and results and we conclude in Section V.

\section{RELATED WORK}

Earlier works on routing and wavelength assignment in optical networks considered ideal physical layer conditions [11]-[13] and thus, the regeneration placement was not taken into account. Nowadays, even with the advance in the fibre technologies, current line rates of $40 \mathrm{~Gb} / \mathrm{s}$ and $100 \mathrm{~Gb} / \mathrm{s}$ with over 160 wavelengths [14] demand high transmission energy 
which pose a serious limitation on the distance a signal can travel without regeneration.

Both linear and nonlinear impairments have been defined and modeled (cf. [15]) and several multiple Impairment Aware RWARP (IA-RWARP) approaches have been studied [6]-[10]. A good survey on the subject may be found in [16]. As the impairment constraints highly depend on the network architecture [15] (fibre characteristics, transmission and switching equipment, channel speed, etc.), studies often simplify these constraints to a single impairment metric [3]-[5] where the terms Quality of Transmission (QoT) and Optical Reach $(\mathcal{R})$ are used as metric. The QoT is often a function of the linear impairments added to an overestimation of the nonlinear impairments whereas the Optical Reach is the maximum distance a signal can travel without regeneration. In [5], Beshir and Kuipers et al. stated that for regenerator placement problems, single impairment metric representing the worst impairment among all the impairments on a link suffices. They also note that the distance may be also used as a single metric as it is a good indicator of the signal quality.

The IA-RWARP approaches have two main variants. The first variant, which was taken as a first approach [10], [17], [18], is to find feasible routes for the demands while minimizing the number of regeneration nodes (where a node can have several regenerators). On the other hand, the second variant focus on minimizing the number of regenerators [3], [5][9]. In [3], Rahman et al. demonstrated intuitively that the impairment aware routing in optical networks should focus on minimizing the number of regenerators rather than minimizing the number of nodes with regeneration as by doing so, the total number of regenerators can be minimal.

\section{RWARP PROBLEM}

Throughout this section we will focus on the WDM Impairment Aware Routing and Wavelength Assignment and Regenerator Placement (IA-RWARP) problem which we will simply call RWARP. Given a network with single fibre links (in fact, each link will have a fibre pair to support traffic in both directions) and a static set of demands, the objective is to route each request through the network by allocating feasible lightpaths while minimizing the number of required regenerators. Bidirectional traffic will be considered and we will assume that each demand fully occupies a single wavelength on each link. That is, a transparent lightpath (or simply, a lightpath) will be a segment of a single end-to-end path for a given request. Of course, if no regeneration is required, the end-toend path will coincide with one single lightpath. We will also assume that every fibre has the same number of wavelengths. Furthermore, the metric used for the impairment of each link is the length that an unregenerated segment can have (with a maximum of $\Delta$ kilometres, where $\Delta$ is the optical reach). The regeneration will be per wavelength - selective regeneration and each regenerator is capable to shift the input wavelength to any output wavelength - wavelength conversion capability.

Beshir and Kuipers et al. in [5] provided an exact Integer Linear Programming (ILP) formulation for the RWARP problem with link-disjoint dedicated path protection without wavelength conversion. In this section we present a new formulation based on their work with the following modifications to conform the aforementioned considerations:

- The need for path protection was suppressed;

- Capacity and Bidirectional traffic restrictions were added;

- Replacement of a constraint to ensure that regenerators can introduce wavelength conversion capability in the network.

\section{A. Notation}

Let $\mathcal{G}=(\mathcal{N}, \mathcal{A})$ denote the physical directed network where $\mathcal{N}$ is the set of $n$ nodes composing the graph and $\mathcal{A}$ the set of $m$ arcs connecting the nodes. An arc is referred as an ordered pair $(i, j)$ where $i$ and $j$ are the nodes connected by the arc, with $i, j \in \mathcal{N}$ and $(i, j) \in \mathcal{A} \cdot \mathcal{A}^{+}(i)=(i, j) \in \mathcal{A}: j \in \mathcal{N}$ denotes the set of outgoing arcs from node $i$ and $\mathcal{A}^{-}(i)=$ $(j, i) \in \mathcal{A}: j \in \mathcal{N}$ denotes the set of incoming arcs. The cost of an $\operatorname{arc}(i, j)$ is represented by $c_{i j}$.

In $\mathcal{G}$, a topological path $p$ (or simply a path, when there is no ambiguity) from a source node $s$ to a destination node $d$ is defined as a sequence of nodes $p_{s d}=\left\langle s, u_{0}, u_{1}, \ldots, u_{w-1}, d\right\rangle$ where $s, d, u_{k} \in \mathcal{N}$ for $k=0,1, \ldots, w-1$. The set of nodes in a path $p_{s d}$ shall be referred to as $\mathcal{N}_{p_{s d}}$ and the set of arcs $\mathcal{A}_{p_{s d}}$. A path $p_{s d}$ may also be defined as a concatenation of sub-paths such that $p_{s d}=p_{s u} \diamond p_{u d}$, that is, $p_{s d}$ coincides with path $p_{s u}$ from $s$ to $u$ and with $p_{u d}$ from $u$ to $d$. The number of arcs in $p_{s d}$ shall be called $\left|\mathcal{A}_{p_{s d}}\right|$ and the cost of the path $c\left(p_{s d}\right)=\sum_{(i, j) \in p_{s d}} c_{i j}$. A set of paths is referred to as $P$ where, more specifically, $P_{s d}$ is a set of paths from a node $s$ to a node $d$.

Let $\mathcal{I}$ denote the set of demands where a single demand $i$ is defined by its source node $s_{i} \in \mathcal{N}$ and the destination node $d_{i} \in \mathcal{N}$. The topological end-to-end path of a demand $i$ is referred to as $p_{i, s d}$. The optical path of the same demand $i, p_{i, s d}^{\lambda}$, is defined as a sequence $p_{i, s d}^{\lambda}=\left\langle l p_{s v_{0}}, l p_{v_{0} v_{1}}, \ldots, l p_{v_{w^{\prime}-1} d}\right\rangle$ where $l p_{x y}$ is a lightpath from $x \in \mathcal{N}$ to $y \in \mathcal{N}$ on some wavelength $\lambda_{x y}$. That is, a lightpath is defined as $l p_{x y}=$ $\left(p_{x y}, \lambda_{x y}\right)$ where $p_{x y}$ is the topological path of the lightpath and $\lambda_{x y}$ is the assigned wavelength. The topological path of $p_{i, s d}^{\lambda}$ is simply the concatenation of the paths in the lightpaths belonging to $p_{i, s d}^{\lambda}$, that is $p_{i, s d}=p_{s v_{0}} \diamond p_{v_{0} v_{1}} \diamond \ldots \diamond p_{v_{w^{\prime}-1} d}$.

\section{B. Problem Formulation and Respective Justification}

We present here an ILP formulation which is a modification (removal of protection) and extension (capacity and bidirectional traffic constraints and wavelength conversion capability at the regenerators) of the dedicated-dedicated ILP formulation proposed in [5].

The underlying network graph will be represented by a directed graph. The links will be transformed into two symmetrical arcs. That is, each link $(i, j)$ is replaced by the arcs $l=(i, j)$ and $l^{\prime}=(j, i)$ of equal capacity. Note that as this study focus on the core of the network, the assumptions that $l$ and $l^{\prime}$ have equal capacity and that all demands are bidirectional and symmetric are realistic. 


\section{Indices:}

$\begin{array}{ll}i=0, \ldots, D-1 & \text { Request ID. } \\ u, v=0, \ldots, N-1 & \text { Node ID. } \\ l, l^{\prime}=0, \ldots, L-1 & \text { Arc ID. } \\ \lambda=0, \ldots, W-1 & \text { Wavelength ID. } \\ \mathcal{A}^{-}(u) / \mathcal{A}^{+}(u) & \text { Incoming/outgoing arcs of node } \mathrm{u}\end{array}$

Binary Variables:

$x_{i, l, u, \lambda} \quad$ Is 1 if wavelength $\lambda$ on $\operatorname{arc} l$ is used by demand $i$ and the last regenerator node on the path before getting to arc $l$ is node $u$. Node $u$ can also be the source node.

$\tau_{i, u, v, \lambda} \quad$ Is 1 if the path of demand $i$ has a regenerator at node $u$ immediately followed by a regenerator at node $v$ on wavelength $\lambda$. Node $u$ can also be

\section{Objective:} the source node.

Minimize the total number of regenerators needed on the network:

$$
\sum_{i} \sum_{\lambda} \sum_{u \in \mathcal{N}} \sum_{v \in \mathcal{N} \backslash\{u\}}\left(\tau_{i, u, v, \lambda}\right)
$$

\section{Constraints:}

Flow Conservation constraints:

At the source node of each demand only a single flow (for that request) can leave the node:

$$
\sum_{l \in \mathcal{A}^{+}\left(s_{i}\right)} \sum_{\lambda} x_{i, l, s_{i}, \lambda}=1 \quad \forall i
$$

where $s_{i}$ is the source node of the demand $i$.

For the intermediate nodes of each demand $i$, i.e. nodes that are not the source nor the destination, the incoming flow has to match the outgoing flow regardless of that node having (or not) regenerator for the given demand:

$$
\begin{array}{r}
\sum_{l \in \mathcal{A}^{-}(v)} x_{i, l, u, \lambda}-\sum_{l \in \mathcal{A}^{+}(v)} x_{i, l, u, \lambda}=\tau_{i, u, v, \lambda} \quad \forall i ; \forall \lambda ; \\
\forall v \in \mathcal{N} \backslash\left\{s_{i}, d_{i}\right\} ; \forall u \in \mathcal{N} \backslash\{v\}
\end{array}
$$

where $v$ is the intermediate node and $d_{i}$ is the destination node of demand $i$. Note that $\tau_{i, u, v, \lambda}$ is equal to 1 when wavelength $\lambda$ is used in the segment between $u$ and $v$ (nodes with regeneration or $u$ as source and $v$ with regeneration) which is a transparent lightpath associated with demand $i$.

If a node $v$ has a regenerator used by demand $i$, the last node with a regenerator in the next segment should be node $v$ :

$$
\begin{array}{r}
\sum_{l \in \mathcal{A}^{+}(v)} \sum_{\lambda} x_{i, l, v, \lambda}-\sum_{\substack{u \in \mathcal{N} \backslash\{v\} \\
\forall i ; \forall v \in \mathcal{N} \backslash\left\{s_{i}, d_{i}\right\}}} \sum_{i, u, v, \lambda}=0 \\
\end{array}
$$

$v$ is thus the tail of the next lightpath and the head of the previous lightpath (from $u$ to $v$ ). Note that the sum for all $\lambda$ 's is needed as the outgoing $\lambda$ may be different from the regenerated $\lambda$, as we are assuming that regenerators may be simultaneously wavelength converters. This new constraint (w.r.t. [5]) may be also stated as: if $v$ has a regenerator for wavelength $\lambda$ required by demand $i$ (previously regener- ated/originated at an upstream node $u$ ), then an outgoing flow for demand $i$ must exist on node $v$, using one of the emergent arcs from $v$ and any available wavelength on that arc.

\section{Wavelength constraints:}

At a given arc $l$ one wavelength $\lambda$ may be used, at most, by a single lightpath:

$$
\sum_{i} \sum_{u \in \mathcal{N}} x_{i, l, u, \lambda} \leq 1 \quad \forall l \in \mathcal{A} ; \forall \lambda
$$

\section{Simple Path constraints:}

The end-to-end path should be a simple path, i.e. without cycles. Thus the source node of a given request should not have any incoming flow relative to that request:

$$
\sum_{l \in \mathcal{A}^{-}\left(s_{i}\right)} \sum_{u \in \mathcal{N}} \sum_{\lambda} x_{i, l, u, \lambda}=0 \quad \forall i
$$

Also at the source node and for each demand, the outgoing flow that is not originated on this node should be 0 :

$$
\sum_{l \in \mathcal{A}^{+}\left(s_{i}\right)} \sum_{u \in \mathcal{N} \backslash\left\{s_{i}\right\}} \sum_{\lambda} x_{i, l, u, \lambda}=0 \quad \forall i
$$

For the intermediate nodes case, the incoming flow for a given request $i$ should be either 1 or 0 , that is only one lightpath of a give demand may be incident to the node:

$$
\sum_{l \in \mathcal{A}^{-}(v)} \sum_{u \in \mathcal{N}} \sum_{\lambda} x_{i, l, u, \lambda} \leq 1 \quad \forall v \in \mathcal{N} \backslash\left\{s_{i}\right\} ; \forall i
$$

\section{Impairment constraints:}

Any lightpath must satisfy an impairment threshold of $\Delta$ :

$$
\sum_{l \in \mathcal{A}} \sum_{\lambda} r(l) \cdot x_{i, l, u, \lambda} \leq \Delta \quad \forall u \in \mathcal{N} ; \forall i
$$

with $r(l)$ the distance cost of arc $l$. Note that $r(l)=c_{i j}$, with $l=(i, j)$.

Bidirectional Traffic constraints:

$$
\sum_{i} \sum_{u \in \mathcal{N}}\left(x_{i, l, u, \lambda}+x_{i, l^{\prime}, u, \lambda}\right) \leq 1 \quad \forall l ; \forall \lambda
$$

this constraint assures that if a given wavelength is in use on a given arc the symmetrical arc on the same wavelength can't be used, as it is needed (already in use) by the bidirectional traffic.

\section{Capacity constraints:}

Finally, the capacity constraint must take into account the bidirectional traffic:

$$
\sum_{i} \sum_{u} \sum_{\lambda}\left(x_{i, l, u, \lambda}+x_{i, l^{\prime}, u, \lambda}\right) \leq C_{l} \quad \forall l
$$

where $C_{l}$ is the arc $l$ capacity in terms of number of wavelengths. This constraint states that the traffic that goes through $l$ and $l^{\prime}$ is at most $C_{l}$ as the remaining capacity (recall that a pair of fibres is to be considered) will be needed for the bidirectional traffic. 
Algorithm 1 RWARP heuristic

Input: A Graph $\mathcal{G}(\mathcal{N}, \mathcal{A})$, an impairment threshold $\Delta$ and an array of demands $\mathcal{I}$

Output: $P_{\mathcal{I}}^{\lambda}=\left\{p_{i, s d}^{\lambda}: i \in \mathcal{I}\right\}$, the set of optical paths $p_{i, s d}^{\lambda}$ where $i$ is an index of a demand from $\mathcal{I}$, and $s$ and $d$ the source and destination of $i$, respectively. A given $p_{i, s d}^{\lambda}$ may be null, due to the lack of capacity in the network

1: $P_{\mathcal{I}}^{\lambda} \leftarrow \emptyset$

2: Sort $\mathcal{I} \triangleright$ By decreasing distance of the corresponding shortest path

3: for $i \in \mathcal{I}$ do

4: $\quad$ For each pair of nodes $u, v \in \mathcal{N}$, compute the shortest path $p_{u v}^{*}$ such that $c\left(p_{u v}^{*}\right) \leq \Delta$ and each $\operatorname{arc} \in A_{p_{u v}^{*}}$ has at least a free wavelength

5: $\quad$ Create a graph $\mathcal{G}^{\prime}\left(\mathcal{N}, \mathcal{A}^{\prime}\right)$ where $\mathcal{A}^{\prime}$ contains the arcs $(u, v)$ where each arc of cost $1+\left(\left|\mathcal{A}_{p_{u v}^{*}}\right| / m\right)$ logically represents a path $p_{u v}^{*}$

6: $\quad$ Find all paths of minimum cost $p_{s d}^{\prime k}$ in $\mathcal{G}^{\prime}$, where $k$ is used as an index for these paths. If no path was found, increment $i$ and go to step 4

7: $\quad$ Expand the sequence of arcs of $p_{s d}^{\prime k}$ to the corresponding sequence of paths $p_{u v}^{*}$ in $\mathcal{G}$ to obtain $p_{s d}^{k}$

8: $\quad$ Remove possible existing loops in all $p_{s d}^{k}$ paths

9: $\quad$ Remove identical paths, if any, from the paths obtained in step 8 to obtain $\hat{P}_{s d}^{k}=\left\{p_{s d}^{k}, k_{s d}=1, \ldots, K_{s d}\right\}$ where $K_{s d}$ is the number of the remaining paths

10: $\quad p_{i, s d}^{\lambda} \leftarrow$ tryFastWavelengthAssignment $\left(\hat{P}_{s d}^{k}\right)$

11: $\quad$ if $p_{i, s d}^{\lambda}=$ null then $\triangleright$ Wavelength assignment failed

12: $\quad p_{i, s d}^{\lambda} \leftarrow$ assignWavelength $\left(\hat{P}_{s d}^{k}\right)$

13: $\quad$ end if

14: $\quad P_{\mathcal{I}}^{\lambda} \leftarrow P_{\mathcal{I}}^{\lambda} \cup\left\{p_{i, s d}^{\lambda}\right\} \triangleright$ New path added to solution set 15: end for

16: return $P_{\mathcal{I}}^{\lambda}$

\section{Heuristic Formulation}

Algorithm 1 illustrates the RWARP heuristic in pseudo code where conceptually three main parts may be observed: demand selection (steps 2 and 3), routing for the selected demand (steps 4 to 9) and finally the wavelength assignment and regenerator placement for each path (steps 10 to 13). Starting at the demand selection, step 2 sorts the array of demands according to a predefined criteria. In [3], Rahman et al. observed that for the routing and wavelength assignment the deployment of lightpaths in a longest-route-first provides, in general, better performance when comparing to the shortest-route-first and random selection. As each demand will be satisfied with a set of consecutive lightpaths (and their symmetric), sorting by the longest-route-first will be advantageous to ensure that this higher demanding requests have a free wavelength on each arc. As at step 2 the routes are still unknown, the shortest path from source to destination of each demand is used as an approximation to the distance of the final demand path.
In this work, the shortest path from a source to a destination in a given network was computed with Dijkstra shortest path algorithm implemented with a binary heap. After sorting the demands in $\mathcal{I}$, the heuristic proceeds to find a feasible path from source to destination for each demand sequentially (steps 4 to 15). In step 4 a set of shortest paths from each node $u$ to every node $v$ that have at least a free wavelength (enough capacity for $i$ ) and with a cost (impairment value) below or equal to the threshold is obtained. This set of paths is used in step 5 to create an auxiliary graph $\mathcal{G}^{\prime}\left(\mathcal{N}, \mathcal{A}^{\prime}\right)$ where $\mathcal{A}^{\prime}=\left\{(u, v): c\left(p_{u v}^{*}\right)<\Delta, A_{p_{u v}^{*}} \subset \mathcal{A}\right\}$, that is, $\mathcal{A}^{\prime}$ contains logical arcs that connect the source $u$ to the destination $v$ of each path obtained in step 4. These logical arcs are set to have a cost of 1 (a hop) plus the division of the number of arcs in the path $p_{u v}^{*},\left|\mathcal{A}_{p_{u v}^{*}}\right|$, by $m$ (the number of arcs in $\mathcal{G}$ ). This cost value will always be greater than 1 by an increment that depends on the number of physical arcs in the path. This increment discriminates longer paths with the same number of logical hops which consequently may conduce to a better overall network capacity usage. Note that the floor of the sum of the cost of any path from $s$ to $d$ in $\mathcal{G}^{\prime}$ will always equal the number of segments in the path and subtracting 1 to this number will equal the number of regenerators needed if no wavelength assignment was to be considered. Using $\mathcal{G}^{\prime}$, all minimum cost paths from $s$ to $d$ are computed in step 6 of algorithm 1 to form the paths $p_{s d}^{\prime k}$ where $k$ is an index to these paths. In this work, the $K$ shortest paths were computed with Ernesto Martins's and Marta Pascoal's implementation of the Yen's $K$ shortest path algorithm [19], as presented in [20]. If no path was found at this step, then there's a capacity shortage and no path will be assigned to the demand, the heuristic will thus return to the beginning of the cycle at step 4 for the next demand. Step 7 transforms/maps the logical arcs in $p_{s d}^{\prime k}$ to the corresponding $p_{u v}^{*}$ in $\mathcal{G}$ producing the real end-to-end paths $p_{s d}^{k}$. At this step loops may arise. Step 8 removes these loops but may consequently create identical paths which are then removed at step 9 to form $\hat{P}_{s d}^{k}-$ the set of candidate paths. The final path - optical path - is selected from the set of candidate paths either in algorithm 2 (tryFastWavelengthAssignment()) or in algorithm 3 (assignWavelength () ) where the wavelength assignment and regenerator placement take place. The former algorithm is faster but may fail often when the network starts to have low spare capacity whereas the latter algorithm will always provide a solution when called from the RWARP heuristic and will thus be called in case of failure of the former. Both algorithms are responsible for creating the lightpaths, assigning the wavelength and placing the respective regenerators and thus, at the end of step 13 an optical path $p_{i, s d}^{\lambda}$ was successfully computed. Having $p_{i, s d}^{\lambda}$, computing the inverse optical path $\left(p_{i, d s}^{\lambda}\right)$ is as simple as using the same wavelengths in the symmetric arcs of $p_{i, s d}^{\lambda}$. The regenerators are considered bidirectional and thus are already computed.

Note that this heuristic is an extension and modification of the Exact Single Request Regenerator Placement (ESRRP) - 
Algorithm 2 tryFastWavelengthAssignment

Input: A set of paths $\hat{P}_{s d}^{k}$ and an impairment threshold $\Delta$

Output: An optical path $p_{s d}^{\lambda}$. The output path is the first element of $\hat{P}_{s d}^{k}$ for which a successful wavelength assignment was possible; if the wavelength assignment failed for every path in $\hat{P}_{s d}^{k}$ then the output is a null path

1: Sort $\hat{P}_{s d}^{k} \stackrel{\triangleright}{s d}$ Sorted according to non-decreasing distance

2: for $p_{s d} \in \hat{P}_{s d}^{k}$ do

3: $\quad$ Create consecutive max possible distance segments from $s$ to $d\left(p_{s u_{0}}, p_{u_{0} u_{1}}, \ldots p_{u_{k-1} d}\right)$ respecting $\Delta$ and try to assign a wavelength to each of these segments. Let $l p_{w}=\left(p_{w}, \lambda_{w}\right), \forall w \in\left\{s u_{0}, u_{0} u_{1}, \ldots, u_{k-1} d\right\}$ if a wavelength was successfully assigned to a $p_{w}$ or null otherwise

4: $\quad$ if $l p_{w} \neq$ null, $\forall w$ then

5: $\quad p_{s d}^{\lambda} \leftarrow\left\langle l p_{s u_{0}}, l p_{u_{0} u_{1}}, \ldots, l p_{u_{k-1} d}\right\rangle$

6: $\quad$ Place regenerators at the last node of each lightpath $l p_{w} \forall w \neq u_{k-1} d$, for the assigned wavelength $\lambda_{w}$ 8: $\quad$ end if

9: end for

10: return null $\triangleright$ No wavelength assignment was possible

algorithm 4 in [6]. The initial base steps were based in the ESRRP but the following changes were introduced: First, we ensure that in step 4, each arc in the shortest path $p_{u v}^{*}$ has a free wavelength. As we are considering capacity constraints, an arc may have a capacity shortage turning it unusable for the next demands. Second, the cost of the arcs of graph $\mathcal{G}^{\prime}$ (see step 5 of algorithm 1) are not equal to one, as in ESRRP. Finding the shortest path in $\mathcal{G}^{\prime}$ will not only provide the minimum number of regenerators but also the path that requires less physical hops (in $\mathcal{G}$ ) among the paths that require minimal regeneration. We then used Yen's algorithm [20] to obtain a set of alternative shortest paths instead of a single one which allows to explore the wavelength assignment on multiple optimal paths (w.r.t. the number of regenerators). Finally, the wavelength assignment and regeneration placement is made so that the wavelength continuity constraint is respected in unregenerated segments. Without capacity constraints, there's always a free wavelength that can be use in any segment and thus this step is not required. This is not the case in this work. Thus, we attempt to assign wavelengths taking only into account the distance of the segments in step 10 (see algorithm 2). If that fails due to wavelength continuity constraints, the regenerators placement is re-examined, in step 12 (see algorithm 3) in order to obtain a solution.

The tryFastWavelengthAssignment() routine starts by sorting the input set of paths $\hat{P}_{s d}^{k}$ accordingly to the path distances. The shortest path will be the path requiring less regeneration (recall that distance is the impairment taken into account) so that every lightpath has a distance as close to $\Delta$ as possible. Starting with the most favourable path, in step 3 , ligthpaths are attempted to be formed by creating maxi- mum distance (unregenerated) segments with respect to the impairment threshold and then assigned a wavelength to each of these segments. Wavelength assignment was implemented using a First-Fit (FF) approach [11]. For efficiency purposes, each arc contains an array of bits where each index represent a wavelength and the value of 1 represents a free wavelength. To obtain the FF free common wavelength on a segment, consecutive and operations through the arcs of the segment are executed and at the end, the index of the first bit with a value of 1 is returned. Note that as the regenerators have wavelength conversion capabilities and at the end of the segments a regenerator is needed, each segment may have a different wavelength but a lightpath in the segment has to comply to the wavelength continuity constraint in all of its composing arcs. The arcs belonging to a given segment may have different free wavelengths and thus finding a common free wavelength to assign to the segment may fail. If wavelength assignment failed in step 3 of algorithm 2, the for loop proceeds to the next favourable path and if all paths fail, the method shall return a null path - step 10. If all segments of a path are successfully assigned a wavelength - forming the respective lightpaths - the optical path $p_{s d}^{\lambda}$ is thus the sequence of these lightpaths from $s$ to $d$ in step 5. The routine continues at step 6 by placing regenerators for the assigned wavelength at the head of each lightpath except at the last head as it will be $d$, the destination node. Step 7 returns the formed path.

As one can see, the tryFastWavelengthAssignment() routine prioritizes the distance when creating the segments without taking into account if there's a free common wavelength at each arc of a segment (albeit having at least a free wavelength on each arc). This may fail often when there's a shortage of free capacity on the arcs as the demands start to occupy some arcs in the network. The routine assignWavelength $($ ) (see algorithm 3) on the other hand tries to create the longest segments on a free common wavelength, respecting the impairment distance threshold. The tryFastWavelengthAssignment() routine provides the same number of regenerators as the assignWavelength () when there's enough capacity for the maximum distance segments to be formed. When this is not the case, the former will fail to retrieve a path but the latter will successfully compute one as algorithm 1 ensures that, after step 6, a path with enough capacity does exist. In short, algorithm 2 is faster and will work for the vast majority of cases but will fail for a large number of demands or for poorly capacitated networks whereas algorithm 3 is slower but will always provide a solution. Algorithm 3 ilustrates the assignWavelength() routine described next. For each path $p_{s d}$ in the set of input paths $\hat{P}_{s d}^{k}$, a corresponding path composed of logical arcs will be created and added to the set of logical paths $P_{s d}^{\prime}$ in steps 1 to 5. A logical path $p_{s d}^{\prime}$ is created using a reachability graph $\mathcal{G}^{\prime \prime}\left(\mathcal{N}_{p_{s d}}, \mathcal{A}^{\prime \prime}\right)$ where $\mathcal{N}_{p_{s d}}$ is the set of nodes in the corresponding $p_{s d}$ and $\mathcal{A}^{\prime \prime}$ is a set of logical arcs of cost 1 from every $u \in \mathcal{N}_{p_{s d}}$ to every downstream node $v \in \mathcal{N}_{p_{u d}} \backslash\{u\}$, with $p_{s d}=p_{s u} \diamond p_{u d}$, at a distance lower or equal to the impairment threshold and have at least a free 
Algorithm 3 assignWavelength

Input: A set of paths $\hat{P}_{s d}^{k}$

Output: An optical path $p_{s d}^{\lambda}$. The output path is the first element of $\hat{P}_{s d}^{k}$ for which a successful wavelength assignment was possible; if the wavelength assignment failed for every path in $\hat{P}_{s d}^{k}$ then the output is a null path.

1: Set $P_{s d}^{\prime} \leftarrow \emptyset$ where $P_{s d}^{\prime}$ is an auxiliar set of logic paths from $s$ to $d$

2: for each $\left(p_{s d} \in \hat{P}_{s d}^{k}\right)$ do

3: $\quad$ Create a reachability graph $\mathcal{G}^{\prime \prime}\left(\mathcal{N}_{p_{s d}}, \mathcal{A}^{\prime \prime}\right)$ where $\mathcal{A}^{\prime \prime}$ is a set of logical arcs with a cost of 1 (hop) from every $u \in \mathcal{N}_{p_{s d}}$ to every $v \in \mathcal{N}_{p_{u d}} \backslash\{u\}$, with $p_{u d}$ the remaining segment from $u$ to $d$ (that is $p_{s d}=$ $p_{s u} \diamond p_{u d}$ ), as long as the segment from $u$ to $v$ has a distance lower than $\Delta$ and there is at least a free common wavelength along the segment

4: $\quad$ Compute the shortest path from $s$ to $d, p_{s d}^{\prime}$ on $\mathcal{G}^{\prime \prime}$ and add it to the set of $P_{s d}^{\prime}$

\section{5: end for}

6: if $P_{s d}^{\prime} \neq \emptyset$ then $\triangleright$ A solution must exist if called from algorithm 1

7: $\quad$ Select the minimum hop count path $p_{s d}^{\prime *}$ from all paths in $P_{s d}^{\prime}$

8: Expand the logic arcs of $p_{s d}^{\prime *}$ into the segments assigning the corresponding free wavelength obtained in step 3 to obtain the lightpaths $l p_{w}, \forall w \in\left\{s u_{0}, u_{0} u_{1}, \ldots, u_{k-1} d\right\}$. Let $p_{s d}^{\lambda}=$ $\left\langle l p_{s u_{0}}, l p_{u_{0} u_{1}}, \ldots, l p_{u_{k-1} d}\right\rangle$ be the final optical path

9: $\quad$ Place regenerators at the last node of each lightpath $l p_{w}, \forall w \neq u_{k-1} d$, for the assigned wavelength $\lambda_{w}$

11: end if return $p_{s d}^{\lambda}$

12: return null $\triangleright$ If called from the RWARP heuristic in algorithm 1 it won't reach here.

common wavelength in the segment from $u$ to $v$. That is, the $\mathcal{G}^{\prime \prime}\left(\mathcal{N}_{p_{s d}}, \mathcal{A}^{\prime \prime}\right)$ graph connects every node $u$ in path $p_{s d}$ to all reachable (w.r.t. impairment threshold and a free common wavelength of the connecting arcs) downstream nodes. Recall that by assigning a cost of 1 (a hop) to a segment from $u$ to $v$, the cost of a logic path from $s$ to $d$ in $\mathcal{G}^{\prime \prime}$ minus 1 will be the number of regenerators needed to traverse the path. After generating this auxiliary graph in step 3, the shortest path $p_{s d}^{\prime}$ is computed at step 4 as a set of consecutive logic arcs and added to the set of logical paths $P_{s d}^{\prime}$. This logical path creation is repeated for each input path which will then be used to create a final path in steps 7 to 9 . The final path is selected as the minimum hop count path in $P_{s d}^{\prime}$ (see step 7 in algorithm 3). As the objective of the RWARP heuristic is to minimize the number of regenerators, the minimum hop count path will be most suitable path. In step 8 , the logical arcs in $p_{s d}^{\prime *}$ are mapped into the real corresponding segments obtained in step 3 and assigned the respective wavelength to form the lightpaths of the optical path $p_{s d}^{\lambda}$. Step 9 places regeneration as in step 6 of algorithm 2 and the optical path is finally returned at step 10 .

\section{Simulations And Results}

The simulations were performed in four different networks from SNDlib [21]: polska, abilene, nobel_germany and janos_us_ca. As the links in nobel_germany are short, the links distance were doubled, and therefore this network will be denote as nobel_germany*.

Table I presents the comparison results between the ILP and the Heuristic. Columns 1, 2, 3 and 4 are the input data corresponding to: the network, the number of demands $|\mathcal{I}|^{1}$, the threshold value $\Delta$ and the capacity (number of wavelengths) in each arc $|\lambda|$, respectively. Columns 5 to 10 are the output results where the left value of " $/$ " presents the CPLEX result and the right value is the heuristic result. The outputs are, respectively, from column 5 to 10: the number of fulfilled demands, the number of lightpaths formed, the network capacity usage (in percentage), the maximum number of wavelengths used in any link of the network, the number of regenerators placed and the execution time (in seconds). The network capacity usage is simply the ratio between the total wavelengths in use and the total number of wavelengths in the network (recall that each demand requires one full wavelength on each arc of its path). The CPLEX execution time was limited to 24 hours, where the results with "time out" provided either a sub-optimal solution or no solution at all (marked with "-"). The "killed" time results means that the tests were terminated with an out of memory error. Also note that table I and II were run on different computers. Table I results suggest that our heuristic provides the minimum number of regenerators for the case where no link is used at maximum capacity (values in column "Max in use $\lambda$ " are inferior to $|\lambda|$ ), that is, the shortest path is always the final path. This result is in fact proven by Kuipers et al. in [6], for their algorithm 4 - Exact Single Request Regenerator Placement (ESRRP) where similar base steps are taken, without taking capacity into account. For the cases where at least one link is at maximum capacity usage (maximum number of wavelengths in use in a link equals $|\lambda|$, the link capacity), our heuristic places slightly more regenerators than the optimal solution ILP except in one case, but in another case, less than the sub-optimal solutions.

Most of the tested networks that timed out at the 24 hours limit providing either a sub-optimal, or no solution at all, took fractions of a second in our heuristic (except for the largest network). Also interesting to note is that by using the shortest path in the heuristic, the network capacity usage is, for most cases, inferior to the ILP solution. This was to be expected as the ILP does not distinguish paths that need the same number of regenerators, but have different number hops. Lastly, note that even for small networks like Polska, when the number of demands is high, the ILP timed out but the heuristic takes less

\footnotetext{
${ }^{1}$ The SNDlib networks contain demands between each pair of nodes. We either used this base set of demands or multiples of them.
} 
TABLE I

COMPARISON BETWEEN ILP AND HEURISTIC

\begin{tabular}{|c|c|c|c|c|c|c|c|c|c|}
\hline \multicolumn{4}{|c|}{ Input data } & \multicolumn{6}{|c|}{ CPLEX / Heuristic results } \\
\hline Network & $|\mathcal{I}|$ & $\Delta$ & $|\lambda|$ & $\begin{array}{c}\text { Fulfilled } \\
\text { Demands }\end{array}$ & $\begin{array}{c}\text { Lighpaths } \\
\text { Formed }\end{array}$ & $\begin{array}{c}\text { Capacity } \\
\text { Usage }(\%)\end{array}$ & $\begin{array}{c}\text { Max in } \\
\text { Use } \lambda\end{array}$ & $\begin{array}{l}\text { No. Reg. } \\
\text { Placed }\end{array}$ & Time(s) \\
\hline \multirow{4}{*}{ polska } & 66 & 1000 & 48 & $66 / 66$ & $134 / 134$ & $17.94 / 16.67$ & $16 / 15$ & $1 / 1$ & $206.47 / 0.14$ \\
\hline & 132 & 1000 & 24 & $132 / 132$ & $268 / 284$ & $66.67 / 71.76$ & $24 / 24$ & $2 / 10$ & $4363.83 / 0.26$ \\
\hline & 132 & 1000 & 48 & $132 / 132$ & $268 / 268$ & $35.76 / 33.33$ & $28 / 30$ & $2 / 2$ & $667.97 / 0.21$ \\
\hline & 264 & 1000 & 48 & $-/ 264$ & $-/ 568$ & $-/ 71.76$ & $-/ 48$ & $-/ 20$ & killed / 0.31 \\
\hline \multirow{4}{*}{ abilene } & 66 & 3000 & 20 & $66 / 66$ & $174 / 180$ & $58.00 / 57.00$ & $19 / 20$ & $21 / 24$ & $606.16 / 0.11$ \\
\hline & 66 & 3000 & 48 & $66 / 66$ & 174 / 174 & $24.44 / 23.47$ & $21 / 22$ & $21 / 21$ & $636.27 / 0.11$ \\
\hline & 132 & 3000 & 40 & $132 / 132$ & $348 / 360$ & $56.83 / 57.00$ & $39 / 40$ & $42 / 48$ & time out / 0.17 \\
\hline & 132 & 3000 & 48 & $132 / 132$ & $348 / 348$ & $47.36 / 46.94$ & $38 / 44$ & $42 / 42$ & $2399.51 / 0.16$ \\
\hline $\begin{array}{c}\text { nobel } \\
\text { germany* }\end{array}$ & 121 & 1000 & 32 & $121 / 121$ & $346 / 330$ & $40.87 / 40.38$ & $31 / 32$ & $52 / 44$ & time out / 0.25 \\
\hline $\begin{array}{l}\text { janos } \\
\text { us ca }\end{array}$ & 1482 & 2000 & 220 & $-/ 1482$ & $-/ 5388$ & - / 51.74 & $-/ 220$ & - / 1212 & killed / 8.24 \\
\hline
\end{tabular}

than one second to find a solution. For larger networks, like janos_us_ca, the heuristic only took about eight seconds and a quarter for solving all demands whereas the CPLEX returned "Out of Memory".

Table II provides a second set of results were the objective was to compare the execution time of four variations of our ILP: ILP-1 has wavelength conversion capabilities at regenerators and capacity constraints (ILP presented in section III); ILP-2 has capacity constraints but no wavelength conversion capabilities (this corresponds to removing the summation in $\lambda$ in equation (4) from ILP-1); ILP-3 does not have capacity constraints (this corresponds to suppressing equation (11) from ILP-1) but has wavelength conversion; ILP-4 is the ILP presented in [5] without path protection, that is, no wavelength conversion neither capacity or bidirectional traffic constraints. The results show that the wavelength conversion capability improves the execution time (ILP-1 to ILP-2). That is to be expected as wavelength conversion relaxes the wavelength continuity constraint. The removal of the capacity constraints also improves the execution time (ILP-1 to ILP-3) as it relaxes the path selection. Note that without capacity constraints, wavelength assignment is irrelevant. Lastly, Beshir's and Kuipers's implementation was the fastest which was to be expected since both capacity and bidirectional constraints were absent.

\section{CONCLUSION}

This work addressed the problem of impairment aware routing and wavelength assignment with regeneration placement (RWARP), considering capacity constraints, with the objective on minimizing the number of regenerators. A previously existing ILP formulation [5] for the RWARP problem was modified and extended to consider capacity constraints, bidirectional traffic and regenerator nodes with wavelength conversion capability. We have also compared some variants of the ILP and concluded that the addition of wavelength
TABLE II

ILP VARIATIONS

\begin{tabular}{|c|c|c|c|c||c|}
\hline \multicolumn{5}{|c||}{ Input data } & CPLEX \\
\hline Network & $|\mathcal{I}|$ & $\Delta$ & $|\lambda|$ & $\begin{array}{c}\text { ILP } \\
\text { Variation }\end{array}$ & Time (s) \\
\hline \multirow{7}{*}{ polska } & 66 & 1000 & 32 & ILP-1 & 180.16 \\
& 66 & 1000 & 32 & ILP-2 & 219.55 \\
& 66 & 1000 & 32 & ILP-3 & 172.15 \\
& 66 & 1000 & 32 & ILP-4 & 108.63 \\
& 132 & 1000 & 48 & ILP-1 & 765.89 \\
& 132 & 1000 & 48 & ILP-2 & 963.53 \\
& 132 & 1000 & 48 & ILP-3 & 781.14 \\
& 132 & 1000 & 48 & ILP-4 & 503.43 \\
\hline
\end{tabular}

conversion capabilities to the network nodes, when capacity constraints are considered, may speed up the ILP. Nevertheless regarding the complexity of the problem, the ILP can only solve small problems in a reasonable amount of time. Therefore we proposed an heuristic which has shown to give accurate results for small networks and is capable of solving large problems in a small amount of time.

In the near future, we intend to extend this work to a multilayer optimization framework considering a MPLS layer over a protected optical network.

\section{ACKNOWLEDGMENT}

Lúcia Martins and Teresa Gomes acknowledge financial support by Fundação para a Ciência e a Tecnologia (FCT) under project grant UID/MULTI/00308/2013. Bruna Nogueira and Ricardo Mendes acknowledge financial support by Coriant Portugal and the University of Coimbra.

\section{REFERENCES}

[1] R. Slavík, F. Parmigiani, J. Kakande, C. Lundström, M. Sjödin, P. a. Andrekson, R. Weerasuriya, S. Sygletos, A. D. Ellis, L. GrünerNielsen, D. Jakobsen, S. Herstrøm, R. Phelan, J. O'Gorman, 
A. Bogris, D. Syvridis, S. Dasgupta, P. Petropoulos, and D. J. Richardson, "All-optical phase and amplitude regenerator for next-generation telecommunications systems," Nature Photonics, vol. 4, no. 10, pp. 690-695, 2010. [Online]. Available: http://www.nature.com/doifinder/10.1038/nphoton.2010.203

[2] M. Matsumoto, "Fiber-based all-optical signal regeneration," IEEE Journal on Selected Topics in Quantum Electronics, vol. 18, no. 2, pp. 738 752, 2012.

[3] Q. Rahman, S. Bandyopadhyay, and Y. Aneja, "On static rwa in translucent optical networks," in Computers and Communications (ISCC), 2012 IEEE Symposium on, July 2012, pp. 000 171-000 176.

[4] — , "A branch and price approach for optimal regenerator placement in translucent networks," in Optical Network Design and Modeling (ONDM), 2011 15th International Conference on, Feb 2011, pp. 1-6.

[5] A. Beshir, F. Kuipers, A. Orda, and P. V. Mieghem, "Survivable routing and regenerator placement in optical networks," in Ultra Modern Telecommunications and Control Systems and Workshops (ICUMT), 2012 4th International Congress on, oct 2012, pp. 684-690.

[6] F. Kuipers, A. Beshir, A. Orda, and P. Van Mieghem, "Impairmentaware path selection and regenerator placement in translucent optical networks," Proceedings - International Conference on Network Protocols, ICNP, pp. 11-20, 2010.

[7] B. Garcia-Manrubia, P. Pavon-Marino, R. Aparicio-Pardo, M. Klinkowski, and D. Careglio, "Offline impairment-aware RWA and regenerator placement in translucent optical networks," Journal of Lightwave Technology, vol. 29, no. 3, pp. 265-277, 2011.

[8] S. Rai, C. F. Su, and B. Mukherjee, "On provisioning in all-optical networks: An impairment-aware approach," IEEE/ACM Transactions on Networking, vol. 17, no. 6, pp. 1989-2001, 2009.

[9] M. A. Ezzahdi, S. Al Zahr, M. Koubàa, N. Puech, and M. Gagnaire, "LERP: A quality of transmission dependent heuristic for routing and wavelength assignment in hybrid WDM networks," Proceedings International Conference on Computer Communications and Networks, ICCCN, pp. 125-136, 2006.

[10] W. Zhang, J. Tang, K. Nygard, and C. Wang, "REPARE: Regenerator placement and routing establishment in translucent networks," GLOBECOM - IEEE Global Telecommunications Conference, 2009.

[11] H. Zang, J. P. Jue, and B. Mukherjee, "A review of routing and wavelength assignment approaches for wavelength- routed optical WDM networks," Optical Networks Magazine, vol. 1, no. January, pp. 47-60, 2000.

[12] Z. Zhang and A. Acampora, "A heuristic wavelength assignment algorithm for multihop wdm networks with wavelength routing and wavelength reuse,' in INFOCOM '94. Networking for Global Communications., 13th Proceedings IEEE, Jun 1994, pp. 534-543 vol.2.

[13] J. M. Yates, M. P. Rumsewicz, and J. P. R. Lacey, "Wavelength converters in dynamically-reconfigurable wdm networks," IEEE Communications Surveys, vol. 2, no. 2, pp. 2-15, Second 1999.

[14] G. Charlet, J. Renaudier, H. Mardoyan, P. Tran, O. B. Pardo, F. Verluise, M. Achouche, A. Boutin, F. Blache, J. Y. Dupuy, and S. Bigo, "Transmission of 16.4-bit/s capacity over $2550 \mathrm{~km}$ using PDM QPSK modulation format and coherent receiver," Journal of Lightwave Technology, vol. 27, no. 3, pp. 153-157, 2009.

[15] J. Strand and A. Chiu, "RFC 4054, impairments and other constraints on optical layer routing," IETF Network Working Group, May 2005. [Online]. Available: http://www.rfc-editor.org/rfc/rfc4054.txt

[16] S. Azodolmolky, M. Klinkowski, E. Marin, D. Careglio, J. S. Pareta, and I. Tomkos, "A survey on physical layer impairments aware routing and wavelength assignment algorithms in optical networks," Computer Networks, vol. 53, no. 7, pp. 926-944, 2009. [Online]. Available: http://dx.doi.org/10.1016/j.comnet.2008.11.014

[17] D. Lucerna, N. Gatti, G. Maier, and A. Pattavina, "On the efficiency of a game theoretic approach to sparse regenerator placement in WDM networks," GLOBECOM - IEEE Global Telecommunications Conference, 2009.

[18] W. Xie, J. P. Jue, X. Wang, Q. Zhang, Q. She, P. Palacharla, and M. Sekiya, "Regenerator site selection for mixed line rate optical networks," IEEE/OSA Journal of Optical Communications and Networking, vol. 6, no. 3, pp. 291-302, March 2014.

[19] J. Y. Yen, "Finding the K Shortest Loopless Paths in a Network," Management Science, vol. 17, no. 11, pp. 712-716, 1971.

[20] E. Q. V. Martins and M. M. B. Pascoal, "A new implementation of Yen's ranking loopless paths algorithm,” 4or, vol. 1, no. 2, pp. 121-133, 2003.

[21] S. Orlowski, R. Wessäly, M. Pióro, and A. Tomaszewski, "SNDlib 1.0 Survivable Network Design library," Networks, vol. 55, no. 3, pp. 276286, 2010, http://sndlib.zib.de. 